\title{
Virus du papillome humain et santé buccodentaire
}

\author{
Bureau du dentiste en chef du Canada ${ }^{1}$
}

\section{Résumé}

Le Canada fait partie des chefs de file mondiaux en santé buccodentaire. Malgré cela, les préoccupations sont de plus en plus vives au sujet des taux croissants de cancers de la bouche et de la gorge liés au virus du papillome humain (VPH). Le lien entre le VPH et le cancer du col de l'utérus est bien établi; heureusement, grâce à la détection et à la vaccination, le Canada a l'un des taux d'incidence les plus faibles du cancer du col de l'utérus au monde. Les cancers de la bouche et de la gorge liés au VPH, cependant, présentent un tableau différent. Au Canada, entre $25 \%$ et $35 \%$ des cancers de la bouche et de la gorge sont liés à l'infection par le VPH par voie orale; et en 2012, le taux d'incidence du cancer de l'oropharynx associé au VPH était plus de 4,5 fois plus élevé chez les hommes que chez les femmes. De plus, la couverture vaccinale contre le VPH au Canada est plus élevée chez les femmes que chez les hommes. Les médecins et les infirmières en santé publique et en milieu clinique ont un rôle à jouer dans la lutte contre la transmission du VPH, tout comme les professionnels de la santé buccodentaire. Les professionnels de la santé buccodentaire peuvent jouer un rôle clé dans la prévention de l'infection par le VPH et des cancers oropharynx liés au VPH en sensibilisant, en éduquant et en offrant des conseils à leurs clients et en encourageant des interventions préventives et diagnostiques fondées sur des données probantes.
Cette oeuvre est mise à la disposition selon les termes de la licence internationale Creative Commons Attribution 4.0

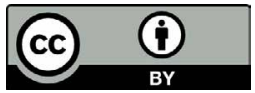

Affiliation

${ }^{1}$ Bureau du dentiste en chef du Canada, Agence de la santé publique du Canada, Ottawa, ON

Correspondance :

claudia.gorenko@canada.ca

Citation proposée : Bureau du dentiste en chef du Canada. Virus du papillome humain humain et santé buccodentaire. Relevé des maladies transmissibles au Canada 2020;46(11/12):427-31.

https://doi.org/10.14745/ccdr.v46i1112a03f

Mots-clés : VPH, cancer de la bouche, sexe oral, sensibilisation, professionnels de la santé buccodentaire

\section{Introduction}

Le Canada est considéré comme l'un des chefs de file mondiaux dans le domaine de la santé buccodentaire (1). La santé buccodentaire est définie par l'Association dentaire canadienne comme étant «l'état des tissus et des structures oraux et connexes qui contribuent positivement au bien-être physique, mental et social et à la jouissance des possibilités de la vie, en permettant à la personne de parler, de manger et de socialiser sans entrave par la douleur, l'inconfort ou l'embarras " (2). Cela pourrait surprendre la plupart des Canadiens que I'on s'inquiète de plus en plus de l'augmentation du nombre de cancers de la bouche et de la gorge liés au virus du papillome humain (VPH) (3). Les infections transmissibles sexuellement (ITS) constituent un important problème de santé publique au Canada (4). Cependant, lorsqu'on pense pour la première fois au sujet des ITS, leur incidence sur la santé buccodentaire n'est souvent pas à l'ordre du jour. L'infection par le VPH est un bon exemple d'une telle connexion négligée. Le VPH est à la fois très courant et très contagieux; et différents types de VPH sont transmis par des activités sexuelles. Plus de $70 \%$ des Canadiens sexuellement actifs seront infectés par le VPH à un moment ou à un autre de leur vie (5). Bien que la plupart des gens contractent ce virus dans leur zone génitale, il peut aussi être contracté dans la bouche et la gorge (3). Les gens ne sont généralement pas au courant de ce fait et des conséquences potentielles d'une infection par le VPH par voie orale (6). Ce survol fournira un résumé du VPH, du cancer de l'oropharynx lié au VPH et de la façon dont les professionnels de la santé buccodentaire peuvent contribuer à réduire le fardeau du cancer de l'oropharynx sur les personnes et les soins de santé.

\section{Épidémiologie du virus du papillome humain}

Il existe plus de 100 types de VPH et le virus peut infecter différentes parties du corps (5). Les souches à faible risque causent des maux mineurs, comme les verrues, tandis que les souches à haut risque peuvent causer le cancer (7). Le VPH est I'infection sexuellement transmissible la plus courante au Canada et dans le monde, et la plupart des Canadiens sexuellement actifs finiront par être infectés par le virus (5). Dans de nombreux cas, l'infection disparaîtra d'elle-même, mais dans une petite partie des cas, où l'infection demeure, elle peut conduire au développement de cancers du col de l'utérus, du vagin, du 
pénis, de l'anus, de la bouche ou de la gorge (8). II faut parfois des années avant qu'une infection par la forme persistante du virus à haut risque ne se transforme, dans certains cas, en cancer. Il est donc important de prévenir la transmission et de vacciner les préadolescents, les adolescents, les jeunes adultes et d'autres groupes potentiellement vulnérables (9).

La relation causale entre le VPH et le cancer du col de l'utérus est bien établie (10). Le VPH est la cause de presque tous les cancers du col de l'utérus (11). En effet, selon un article récent, " le cancer du col de l'utérus continue d'être un problème de santé publique majeur affectant les femmes d'âge moyen, en particulier dans les pays moins dotés en ressources » (12). Selon I'Organisation mondiale de la Santé, le cancer du col de l'utérus est le quatrième cancer le plus fréquent chez les femmes dans le monde (13). Au Canada, cependant, nous avons constaté une forte baisse de l'incidence et de la mortalité au fil du temps, l'un des taux d'incidence les plus faibles du cancer du col de l'utérus au monde (14). La combinaison d'une adoption précoce de tests de dépistage à grande échelle et de l'introduction du vaccin contre le VPH a joué un rôle clé dans ce déclin (15).

Alors que les femmes ont vu des taux décroissants de cancer du col de l'utérus, l'incidence d'autres infections et cancers liés au VPH, y compris le cancer de l'oropharynx, particulièrement chez les hommes, est en hausse (7). Cela est conforme aux observations faites aux États-Unis et dans certains pays européens (7). Tel que présenté au tableau 1, le cancer de I'oropharynx représente le plus grand nombre de cas de cancer liés au VPH au Canada (7). Le cancer de l'oropharynx lié au VPH est principalement causé par la souche VPH-16. La prévalence la plus élevée du VPH est observée chez les adultes de 20 à 24 ans (16), avec $10 \%$ à $30 \%$ des infections actives (17). Au Canada, en 2012, deux tiers de tous les cancers associés au VPH ont été diagnostiqués chez les femmes et un tiers chez les hommes (7). Le tableau 2 ci-dessous présente l'incidence du cancer de l'oropharynx lié au VPH chez les hommes au Canada et aux États-Unis $(17,18)$. Bien que des données comparables pour les mêmes périodes ne soient pas disponibles, on peut voir par ces chiffres que l'incidence augmente.

Tableau 1 : Cancer du virus du papillome humain le plus répandu au Canada, 2012

\section{\begin{tabular}{l|l} 
Type de cancer lié au VPH & Nombre total de cas
\end{tabular}}

\begin{tabular}{|l|r|}
\hline Oropharynx & 1335 \\
\hline Cervical & 1300 \\
\hline Anal & 475 \\
\hline
\end{tabular}

Abréviation : VPH, virus du papillome humain

Sans vaccination, il est probable que la plupart des Canadiens sexuellement actifs seront infectés par le VPH à un moment ou à un autre de leur vie. Malheureusement, de bonnes données épidémiologiques font défaut parce que le VPH n'est pas une maladie à déclaration obligatoire à l'échelle nationale, qu'il
Tableau 2 : Incidence du cancer de l'oropharynx lié au virus du papillome humain au Canada chez les hommes

\begin{tabular}{|c|c|c|}
\hline \multirow{2}{*}{ Année } & \multicolumn{2}{|c|}{ Nombre de cas par 100000} \\
\hline & Canada & États-Unis \\
\hline 1997 & 4,1 & s.o. \\
\hline 2012 & 6,4 & s.o. \\
\hline 2013-2017 & s.o. & 8,7 \\
\hline 2017 & s.o. & 8,9 \\
\hline
\end{tabular}

est généralement asymptomatique et que les diagnostics du VPH ne sont pas disponibles ou financés par le public $(16,19)$. La transmission du VPH oral se fait habituellement par le sexe oral, mais il faut encore poursuivre les recherches pour mieux comprendre s'il existe d'autres modes de transmission orale possibles; et de déterminer quels sont les mécanismes par lesquels, dans certains cas, le virus contribuera au développement du cancer de la bouche et de la gorge (20). De plus, une personne infectée par le VPH par voie orale peut être asymptomatique pendant de nombreuses années, ce qui rend très difficiles la détection et la prévention de la transmission ultérieure (20). Le cancer de l'oropharynx affecte le tiers postérieur de la langue, des amygdales et de la paroi médiale du pharynx et est généralement diagnostiqué à des stades avancés (21).

\section{Vaccin contre le virus du papillome humain}

Alors que les hommes peuvent maintenant recevoir le vaccin contre le VPH, I'accent qui a été mis initialement sur la prévention du cancer du col de l'utérus et l'introduction d'un vaccin exclusivement pour les femmes semble avoir créé un préjugé sexiste qui a conduit à l'idée fausse que le VPH est un "problème de femmes » (22). Toutes les provinces et tous les territoires ont annoncé ou introduit des programmes d'immunisation contre le VPH pour les filles dans le cadre des programmes d'immunisation systématique (23). Cependant, ce n'est qu'en 2017 que toutes les provinces et tous les territoires du Canada ont offert des programmes gratuits d'immunisation scolaire pour le VPH aux garçons et aux filles avec des critères d'admissibilité différents (24-27). Par conséquent, la couverture vaccinale contre le VPH au Canada est probablement plus élevée chez les femmes que chez les hommes (28). Malheureusement, les données détaillées sur la couverture vaccinale au Canada ne sont pas uniformes dans toute la documentation. II faut améliorer la recherche et la surveillance dans ce domaine (28).

Trois États américains, l'Illinois, le Minnesota et l'Oregon, autorisent la vaccination contre la grippe dans les cabinets dentaires (29); cependant, seul l'Oregon autorise également la vaccination contre le VPH dans les cabinets dentaires (29). Les dentistes canadiens n'ont pas actuellement l'autorité 
réglementaire nécessaire pour administrer les vaccins contre le VPH, et la responsabilité incombe aux médecins et aux infirmières pour le moment. Cependant, les vaccinations contre le VPH dans les cabinets dentaires pourraient contribuer à augmenter les taux de vaccination, en particulier chez les hommes. Les considérations relatives aux vaccinations dans les cabinets dentaires canadiens devraient être évaluées et discutées avec les organismes de réglementation compétents. Ces discussions porteraient notamment sur la formation, la détermination de l'existence ou non d'antécédents médicaux suffisants chez les dentistes et l'estimation des coûts connexes (29). Entre-temps, I'administration des vaccinations dans les cabinets dentaires américains devrait faire l'objet d'un suivi afin d'informer toute initiative de ce genre au Canada.

\section{Prochaines étapes}

Avec l'augmentation du cancer de l'oropharynx associé au $\mathrm{VPH}$, il est nécessaire de prendre davantage de mesures pour réduire cette tendance. S'il n'est pas pris en compte, le cancer de l'oropharynx associé au VPH pourrait avoir une incidence importante sur le système de santé et les ressources (7). Les cancers de l'oropharynx sont un problème de santé publique parce qu'ils ont une incidence importante sur les niveaux individuel, social et du système de soins de santé (21). La participation d'un plus grand nombre de garçons aux programmes de vaccination contribuerait à assurer une protection équitable des hommes contre les maladies liées au VPH (22). Étant donné la lenteur de la latence entre l'infection au VPH et le cancer, il se peut que plusieurs années passent avant que l'incidence de la vaccination puisse être évaluée. De plus, il existe des preuves solides que la vaccination des femmes peut aider à prévenir l'infection chez les hommes par l'immunité collective (7). La vaccination de la population avant qu'elle ne devienne sexuellement active est essentielle à la réduction de ce fardeau (5). Il est également nécessaire d'ajouter et de renforcer les messages autour 1) des pratiques et des comportements sexuels, 2) l'importance de la santé buccodentaire dans le cadre de la santé globale et 3 ) du ou des rôles joués par les professionnels de la santé buccodentaire dans la détection des signes précoces d'anomalies dans la bouche (6). Les professionnels de la santé publique doivent continuer à surveiller les tendances changeantes et évolutives des taux de transmission et de vaccination du VPH, et veiller à ce que les tendances observées soient fondées sur le sexe et le genre, étant donné que, par exemple, les hommes sont plus susceptibles de développer des cancers de l'oropharynx que les femmes, tout en étant moins susceptibles de se faire vacciner $(14,22)$.

Les professionnels de la santé buccodentaire peuvent jouer un rôle clé dans la lutte contre la transmission du VPH, en particulier contre l'infection orale par le VPH, et dans la prévention des cancers de l'oropharynx liés au VPH - après tout, I'infection par le VPH peut être évitée - en sensibilisant, en éduquant et en offrant des conseils à leurs clients et en encourageant des interventions préventives et diagnostiques fondées sur des données probantes $(6,13)$. Une attention particulière est nécessaire dans le domaine de la prévalence de l'infection orale au VPH et de ses voies typiques de transmission, outre les tendances de la vaccination. Son rôle dans l'apparition de cancers de la bouche liés au VPH devrait faire l'objet $d$ 'une surveillance et d'une recherche accrues, et il faudrait également poursuivre la recherche afin d'examiner la mauvaise santé buccodentaire, y compris la maladie parodontale, et la mauvaise hygiène buccodentaire en tant que facteurs de risque indépendants pour l'infection au VPH et le cancer de la bouche (30). Une petite étude préliminaire dans ce domaine a indiqué que la capacité des dentistes de l'Ontario de détecter et de prévenir les cancers de la bouche est limitée en raison d'une formation inadéquate (21), tandis qu'une autre petite étude en Floride a montré que les dentistes étaient aux étapes de la contemplation préalable et de la contemplation de l'état de préparation pour discuter des vaccins contre le VPH avec les patients (31). À la lumière de ces études, les professionnels de la santé buccodentaire devraient être encouragés à faire ce qui suit :

- Demeurer au courant des nouvelles données relatives à I'infection par le VPH et aux cancers de la bouche

- Effectuer le dépistage du cancer de la bouche lors des examens cliniques

- Reconnaître et détecter les signes et symptômes à un stade précoce, et surveiller toute lésion anormale ou suspecte dans la bouche

- Étudier la possibilité de prélever des échantillons au bureau dentaire (e.g. rinçage ou écouvillons par voie orale) pour la détection du VPH

- Expliquer aux clients les liens entre le VPH oral et le cancer de la bouche

- Partager des renseignements clairs et fondés sur des données probantes et discuter avec leurs clients des facteurs de risque connus (comme l'usage du tabac) et des modes de transmission, y compris les pratiques et les comportements sexuels

- Continuer de promouvoir activement l'importance d'une bonne hygiène buccodentaire et d'une bonne santé buccodentaire en tant que facteurs de prévention de I'infection au VPH et des cancers de la bouche liés au VPH

- Promouvoir le vaccin contre le VPH comme moyen sûr et efficace de prévenir l'infection

- Discuter avec les organismes de réglementation des dentistes de la possibilité d'administrer le vaccin contre le VPH dans les cabinets dentaires 


\section{Conclusion}

Le lien entre le VPH et le cancer de l'oropharynx est évident; et avec l'augmentation des taux d'incidence, il faut prendre davantage de mesures pour enrayer cette tendance. Les professionnels de la santé buccodentaire peuvent contribuer de nombreuses façons à réduire les taux d'infection par le VPH par voie orale et à prévenir les cancers de l'oropharynx liés au VPH. Les professionnels de la santé buccodentaire sont des acteurs clés dans la lutte contre la transmission du VPH et la prévention du cancer de l'oropharynx et devraient élaborer et mettre en œuvre des plans à l'appui de cette lutte pour la santé et le bien-être de leurs patients.

\section{Déclaration des auteurs}

Aucune.

\section{Intérêts concurrents}

Aucune.

\section{Remerciements}

Le mandat du Bureau du dentiste en chef du Canada est de faire progresser la santé buccodentaire de la population par la promotion de la santé, la prévention des maladies et l'orientation professionnelle et technique, en mettant l'accent sur les populations vulnérables.

Les auteurs aimeraient souligner la contribution de la Bibliothèque de la santé de Santé Canada à la recherche documentaire.

\section{Financement}

Aucune.

\section{Références}

1. Canadian Dental Association. The State of Oral Health in Canada. Ottawa (ON): CDA; 2017. https://www.cda-adc.ca/ stateoforalhealth/_files/TheStateofOralHealthinCanada.pdf

2. Association dentaire canadienne. Énoncés de position de I'ADC. Ottawa (ON) : ADC; 2020. https://www.cda-adc.ca/fr/ about/position_statements/

3. Société du VPH et cancer. 2020 (accédé 2020-06-11). https://www.cancer.ca/fr-ca/prevention-and-screening/ reduce-cancer-risk/make-informed-decisions/get-vaccinated/ hpv-and-cancer/?region=on
4. Agence de la santé publique du Canada. Rapport sur les infections transmissibles sexuellement au Canada, 2017. Ottawa (ON) : ASPC; 2019 (accédé 2020-06-25). https:// www.canada.ca/en/public-health/services/publications/ diseases-conditions/report-sexually-transmitted-infectionscanada-2017.html

5. Agence de la santé publique du Canada. Santé sexuelle et infections transmissibles sexuellement: Virus du papillome humain (VPH). Ottawa (ON) : ASPC; 2020 (accédé 2020-06-11). https://www.canada.ca/ $\mathrm{fr} /$ sante-publique/services/maladies-infectieuses/ sante-sexuelle-infections-transmissibles-sexuellement/ virus-papillome-humain-vph.htm

6. Brondani MA, Siqueira AB, Alves $C M$. Exploring lay public and dental professional knowledge around HPV transmission via oral sex and oral cancer development. BMC Public Health 2019;19(1):1529. DOI PubMed

7. Comité consultatif des statistiques canadiennes sur le cancer. Comité consultatif de la Société canadienne du cancer : Statistiques canadiennes sur le cancer 2016. Toronto (Ontario) : Société canadienne du cancer, 2016; 2016. https://www.cancer.ca/ /media/cancer.ca/CW/cancer\%20 information/cancer\%20101/Canadian\%20cancer\%20 statistics/Canadian-Cancer-Statistics-2016-FR.pdf?la=en

8. Agence de la santé publique du Canada. Maladies et affections : Virus du papillome humain (VPH). Santé Canada : 2017 (accédé 2020-06-11).

https://www.canada.ca/fr/sante-publique/services/maladies/ virus-papillome-humain-vph.htm

9. National Cancer Institute. HPV and Cancer. NCl; January 10, 2020. https://www.cancer.gov/about-cancer/ causes-prevention/risk/infectious-agents/hpv-and-cancer

10. Schiffman $M H$, Castle P. Epidemiologic studies of a necessary causal risk factor: human papillomavirus infection and cervical neoplasia. J Natl Cancer Inst 2003;95(6):e2. DOl PubMed

11. Centers for Disease Control and Prevention (US). HPV and Cancer: Cancers Associated with Human Papillomavirus (HPV). Atlanta (GA): CDC; 2019. https://www.cdc.gov/ cancer/hpv/basic info/cancers.htm

12. Arbyn M, Weiderpass E, Bruni L, de Sanjosé $S$, Saraiya M, Ferlay J, Bray F. Estimates of incidence and mortality of cervical cancer in 2018: a worldwide analysis. Lancet Glob Health 2020;8(2):e191-203. DOI PubMed

13. Organisation mondiale de la Santé. Papillomavirus humain $(\mathrm{PVH})$ et cancer du col de l'utérus. Genève (Suisse) : OMS; 24 janvier 2019. https://www.who.int/fr/news-room/ fact-sheets/detail/human-papillomavirus-(hpv)-and-cervicalcancer 
14. Comité consultatif des statistiques canadiennes sur le cancer. Statistiques canadiennes sur le cancer 2019. Toronto (ON) : Société canadienne de cancer; 2019. https://www. cancer.ca/ /media/cancer.ca/CW/cancer\%20information/ cancer\%20101/Canadian\%20cancer\%20statistics/ Canadian-Cancer-Statistics-2019-FR.pdf?la=fr-CA

15. Steben M, Wurtak G. Canadian Network on HPV Prevention, Canadian Public Health Association, CIDC - Consortium for Infectious Disease Control, HPV Awareness and International Papillomavirus Society. Canada's Role in Accelerating Global Elimination of Cervical Cancer. July 2019 (accessed 2020-06-11). https://static1.squarespace.com/ static/5b855e9a75f9ee482638631e/t/5d1e28aea7584e0001 0b4ef8/1562257586960/cervical-cancer-elim-report_final.pdf

16. Société canadienne de pédiatrie. Le vaccin contre le virus du papillome humain chez les enfants et les adolescents. Document principes. SCP; 12 juin 2018 (accédé 2020-09-08). https://www.cps.ca/fr/documents/position/VPH

17. Train E. HPV-related cancers: Encouraging early diagnosis. Family Practice Oncology CME Day; 2017 Nov 18; Vancouver, Canada. Sept 10, 2020. P. 1-40. http://www. bccancer.bc.ca/family-oncology-network-site/Documents/ TRAN,\%20E.\%20HPV\%20Cancers.pdf

18. Centers for Disease Control and Prevention (US). United States Cancer Statistics: Data Visualizations Tool, based on 2019 submission data (1999-2017). June 2020 (accessed 2020-09-10). https://www.cdc.gov/cancer/dataviz

19. Burd EM. Human papillomavirus and cervical cancer. Clin Microbiol Rev 2003;16(1):1-17. DOI PubMed

20. Syrjänen S. Oral manifestations of human papillomavirus infections. Eur J Oral Sci 2018;126 Suppl 1:49-66. DOI PubMed

21. Aldossri M, Okoronkwo C, Dodd V, Manson H, Singhal S. Dentists' Capacity to Mitigate the Burden of Oral Cancers in Ontario, Canada. J Can Dent Assoc 2020;86:k2. PubMed

22. Shapiro GK, Perez S, Rosberger Z. Including males in Canadian human papillomavirus vaccination programs: a policy analysis. CMAJ 2016;188(12):881-6. DOI PubMed
23. Une déclaration d'un comité consultatif (DCC), Comité consultatif national de l'immunisation (CCNI). Mise à jour sur les vaccins contre le virus du papillome humain (VPH). Relevé des maladies transmissibles au Canada 2012;38(0):1-69. https://doi.org/10.14745/ccdr.v38i00a01f

24. Gouvernement du Québec. Programme de vaccination contre les infections par les virus du papillome humain (VPH). 2020. https://www.quebec.ca/sante/conseils-etprevention/vaccination/programme-de-vaccination-contr e-les-infections-par-les-vph/historique-du-programme/

25. Government of Northwest Territories. Human Papillomavirus (HPV). April 2017. https://www.hss.gov.nt.ca/sites/hss/files/ hpv.pdf

26. Government of Yukon. News Release - Yukon to offer free HPV immunization to boys. August 18, 2017. http://www.hss. gov.yk.ca/17-076.php

27. Government of Nunavut. Safety Information: Gardasil 9. January 2018. https://www.gardasil9.ca/provincial_ programs/nunavut/

28. Bird Y, Obidiya O, Mahmood R, Nwankwo C, Moraros J. Human Papillomavirus Vaccination Uptake in Canada: A systemic review and meta-analysis. Int J Prev Med 2017;8(1):71. DOl PubMed

29. Kwan-Ho Yu J. ADA News- My View: Could vaccines come from your dentist? Not yet. Oct 21, 2019. https:// www.ada.org/en/publications/ada-news/viewpoint/ my-view/2019/october/my-view-could-vaccines-come-fro m-your-dentist-not-yet

30. Dalla Torre D, Burtscher D, Sölder E, Rasse M, Puelacher W. The correlation between the quality of oral hygiene and oral HPV infection in adults: a prospective cross-sectional study. Clin Oral Investig 2019;23(1):179-85. DOI PubMed

31. Daley E, Dodd V, DeBate R, Vamos C, Wheldon C, Kline N, Smith S, Chandler R, Dyer K, Helmy H, Driscoll A. Prevention of HPV-related oral cancer: assessing dentists' readiness. Public Health 2014;128(3):231-8. DOl PubMed 